\title{
Adaptability Analysis of Common Measurement Matrices for Mechanical Vibration Signal
}

\author{
Guo Junfeng \\ School of Mechanical and Electronical Engineering \\ Lanzhou University of Technology \\ Lanzhou, China \\ e-mail: junf_guo@163.com \\ Shi Jianxu \\ School of Mechanical and Electronical Engineering \\ Lanzhou University of Technology \\ Lanzhou, China \\ e-mail: jianx_shi@163.com
}

\author{
Lei Chunli \\ School of Mechanical and Electronical Engineering \\ Lanzhou University of Technology \\ Lanzhou, China \\ Wei Xingchun \\ School of Mechanical and Electronical Engineering \\ Lanzhou University of Technology \\ Lanzhou, China
}

\begin{abstract}
In this paper, the construction method of common measurement matrices is studied, adaptability of common measurement matrices for mechanical vibration signal is analyzed. Typical measurement matrices are selected from commonly used measurement matrices, Gaussian random measurement matrix and Bernoulli random measurement matrix are chosen from totally random measurement matrices, Circulant measurement matrix and Toeplitz measurement matrix are selected from deterministic matrices, partially random Fourier measurement matrix and Hadamard matrix are chosen from deterministic measurement matrices. The sensing performance of common measurement matrices for mechanical vibration signal is evaluated from the two perspective of reconstruction error and memory space. The simulation results show that two kinds of complete random matrices, Gaussian and Bernoulli matrices, can exactly reconstruct original vibration signal, but they occupy large memory space; deterministic matrices, Circulant and Toeplitz matrices, although need fewer memory space, obtained measurements which do not have information of global vibration signal lead to lower reconstruction results; partially random Fourier matrix is extremely coherent with sparse transforming base of vibration signal, so it has not exact result in the process of reconstructing original vibration signal, the requirement of Exponentiation of 2 seriously restrict its application.
\end{abstract}

Keywords-mechanical vibration signal; comprssed sensing; measurement matrices; sparse decomposition; reconstruction algorithm;

\section{INTRODUCTION}

Mechanical vibration signal contains a lot of information of equipment in the work process, on-line monitor, collection and extracting useful information of machinery vibration signal is a key technology in mechanical engineering field, especially in the fault diagnosis and remote fault diagnosis technology ${ }^{[1][2]}$.
Internal mechanism and feature of machine is further mastered, theoretical and technological support of designing and exploiting mechanical equipment is provided via these works. However, with the increasing development of mechanical industry and constantly enhancement of actual manufacturing demand, the trend of machinery equipment in large-scale and complete set is getting inevitable, vibrational frequency is becoming increasingly higher and emerging non-linear, nonstationary since collision, velocity jump, and structural distortion and so on are occurred in the work process. What is more, with the development of mechanical vibration test towards integration, high speed, succession, and networking, the vast amounts of sampling data is generated based on Shannon-Nyquist sampling theory, the real time transmission and synchronous storage of these sampling data are becoming the urgent problems of cost and engineering technology. Compressed Sensing can guarantee the original structure of signal with non-linear projection and apply into sampling of vibrational signal, this can sample vibrational signal with sub-Nyquist rate under the condition of without losing vibrational information ${ }^{[3]}$.

Measurement matrices play a vital role in compressed sampling vibration signal because it is responsible for sampling signal. The performance of measurement matrices is critical factor which can guarantee measurements of containing global information. Null Space Property(NSP) ${ }^{[4]}$, Spark ${ }^{[5]}$, Restricted Isometry Property(RIP) ${ }^{[6]}$, Incoherence ${ }^{[7-10]}$ are the important criteria which describe the performance of measurement matrices. Measurement matrices if only satisfy one of the criteria, the recovery of original signal is achieved by reconstruction algorithms ${ }^{[11]}$. 


\section{THE CONSTRUCTION METHOD OF COMMON MEASUREMENT MATRICES}

\section{A. Gaussian random matrix}

Gaussian random matrix was not only presented firstly in compressed sensing, but also widely used measurement matrix. Its construction process is as follows:

The matrix $\Phi \in R^{M_{\times N}} \quad$ its entries $\left(\varphi_{i, j}(i=1,2, \cdots, M ; j=1,2, \cdots, N)\right)$ obey Gaussian distribution.

Related studies show that Gaussian random measurement matrix satisfies the performance requirement of measurement the matrix.

\section{B. Bernoulli random matrix}

The construction process of Bernoulli random matrix is as follows: generating matrix $\Phi \in R^{M \times N}$, where its entries $\left(\varphi_{i, j}(i=1,2, \cdots, M ; j=1,2, \cdots, N)\right)$ obey Bernoulli distribution, the value of entries is $+/-1$,

$$
\varphi_{i, j}:= \begin{cases}+1 / \sqrt{M} & \text { prob } 1 / 2 \\ -1 / \sqrt{M} & \text { prob } 1 / 2\end{cases}
$$

Because the entries of Bernoulli random matrix have two state, so Bernoulli random matrix is also called binary random matrix.

\section{Partially random Fourier matrix}

First, generating Fourier matrix $W_{N} \in R^{N_{\times N}}$, then randomly selecting $M$ rows from Fourier matrix, finally the selected $M$ rows form measurement matrix $\Phi \in R^{M \times N}$, this forming matrix is called partially random Fourier matrix

$$
W_{N}=\frac{1}{\sqrt{N}}\left[\begin{array}{ccccc}
W^{0} & W^{0} & W^{0} & \cdots & W^{0} \\
W^{0} & W^{1} & W^{2} & \cdots & W^{N-1} \\
W^{0} & W^{2} & W^{4} & \cdots & W^{2(N-1)} \\
\vdots & \vdots & \vdots & \vdots & \vdots \\
W^{0} & W^{N-1} & W^{2(N-1)} & \cdots & W^{(N-1)(N-1)}
\end{array}\right]
$$

Where $W=\exp (-2 \pi i / N), 1 / \sqrt{N}$ denotes normalization factor

Partially random Fourier matrix only is used to sense time sparse signal because partially random Fourier matrix is incoherence with time sparse signal. However, it is extremely rare that the real word signal is time sparse signal. Therefore, the application of partially random Fourier matrix is restricted vastly. In addition, reconstructed mechanical vibration signal is not exact when selecting Fourier matrix as the sparse transform base and partially random Fourier matrix as the measurement matrix based on incoherence theory.

\section{Partially random Hadamard matrix}

First generating Hadamard matrix $H_{N} \in R^{N \times N}$, then randomly selecting $M$ rows from Hadamard matrix, finally the selected $M$ rows form measurement matrix $\Phi \in R^{M \times N}$, this forming matrix is called partially random Hadamard matrix.

\section{E. Circulant matrix}

The type of Circulant matrix is as follows:

$$
\Phi=\left[\begin{array}{ccccc}
a_{N} & a_{N-1} & \cdots & a_{2} & a_{1} \\
a_{1} & a_{N} & \cdots & a_{3} & a_{2} \\
\vdots & \vdots & \ddots & \ddots & \vdots \\
a_{M-1} & a_{M-2} & \cdots & \cdots & a_{M}
\end{array}\right]
$$

Where $\left\{a_{i}\right\}_{i=1}^{N}$ obey Bernoulli distribution.

F. Toeplitz matrix

The form of Toeplitz matrix is as follows:

$$
\Phi=\left[\begin{array}{ccccc}
a_{N} & a_{N-1} & \cdots & a_{2} & a_{1} \\
a_{N+1} & a_{N} & \cdots & a_{3} & a_{2} \\
\vdots & \vdots & \ddots & \ddots & \vdots \\
a_{N+M-1} & a_{N+M-2} & \cdots & \cdots & a_{M}
\end{array}\right]
$$

Where $\left\{a_{i}\right\}_{i=1}^{N}$ obey Bernoulli distribution.

\section{SPARSITY ANALYSIS FOR VIBRATION SIGNAL}

Bearing designation 6205-2RS is studied, the whole feature of roll bearing vibration signal is represented with work frequency, the main doubling frequency and fractional frequency, amplitude vibration and impact vibration is coming into being when existing machinery fault, the vibration signal model of bearing outer ring is established as follows:

$$
\begin{aligned}
f(t)= & \sum_{i=1}^{m} A_{r_{i}} \cos \left(2 \pi i f_{r} t+\varphi_{r_{i}}\right) \\
& +\sum_{j=1}^{n} A_{o_{j}} \cos \left(2 \pi j f_{o} t+\varphi_{o_{j}}\right) \\
& +A_{I} e^{-\alpha t^{\prime}} \cos \left(2 \pi f_{c} t+\varphi_{I}\right)
\end{aligned}
$$

Where $f_{r}$ - rotate frequency

$A_{r_{i}}$ - - amplitude of harmonic vibration

$\varphi_{r_{i}}$ — phase of harmonic vibration

$f_{o}$ — - fault frequency with outer ring

$A_{o_{j}}$ - - amplitude of harmonic vibration with outer ring fault

$\varphi_{o_{j}}$ - phase of harmonic vibration with outer ring fault

$f_{c}$ - carrier frequency with outer ring fault

$A_{I}-$ - amplitude of impose decay vibration with outer ring fault

$\varphi_{I}-$ - phase of impose decay vibration with outer ring fault

$\alpha-$ - decay coefficient

$t^{\prime}=\bmod \left(t, \frac{1}{f_{o}}\right)$ — time of impose decay

Taking bearing designation $6205-2 \mathrm{RS}$ as an example, its inner diameter $d_{1}=25 \mathrm{~mm}$, external diameter $d_{2}=52 \mathrm{~mm} \quad, \quad$ rolling element 
diameter $d_{3}=7.9 \mathrm{~mm}$, contact angle $\beta=0.67 \mathrm{rad}$, rolling element number $z=9$, working frequency $f_{r}=30 \mathrm{~Hz}$, sampling frequency $f_{s}=1024 \mathrm{~Hz}$.So based on the following computational formula of outer ring fault frequency.

$$
f_{o}=\frac{1}{2} \times z \times\left(1-\frac{d_{3}}{d_{2}-d_{1}} \times \cos \beta\right) \times f_{r}
$$

outer ring fault frequency $f_{0}=104.03 \mathrm{~Hz}$ is obtained, when outer ring fault is occurred, supposed carrier frequency $f_{c}=3000 \mathrm{~Hz} . \mathrm{m}=3, \mathrm{n}=5, A_{r i}(i=1,2,3)$, $A_{o j}(j=1,2,3,4,5)$ and $A_{I}$ are $0.1,0.2,0.3,0.3,0.4,0.33,0.2,0$. $1,0.38$,respectively, $\varphi_{r i}(i=1,2,3), \varphi_{o j}(j=1,2,3,4,5)$ and $\varphi_{I} \quad$ are $0,3,2.5,0,2,6,4,4.5,3.3$ respectively, decay coefficient $\alpha=800$.The outer ring vibration model of 6205-2RS is specified as follows:

$$
\begin{aligned}
f(t) & =0.1 \times \cos (2 \pi \times 29.17 \times t)+0.2 \times \cos (2 \pi \times 2 \times 29.17 \times t+3) \\
& +0.3 \times \cos (2 \pi \times 3 \times 29.17 \times t+2.5)+0.3 \times \cos (2 \pi \times 107.75 \times t) \\
& +0.4 \times \cos (2 \pi \times 2 \times 107.75 \times t+2)+0.33 \times \cos (2 \pi \times 3 \times 107.75 \times t+6) \\
& +0.2 \times \cos (2 \pi \times 4 \times 107.75 \times t+4)+0.1 \times \cos (2 \pi \times 5 \times 107.75 \times t+4.5) \\
& +0.38 \times e^{-800 t} \times \cos (2 \pi \times 3000 \times t+3.3)
\end{aligned}
$$

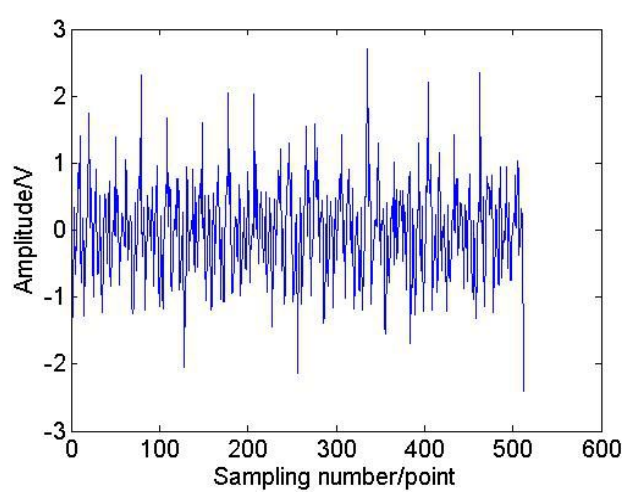

(a) time domain waveform

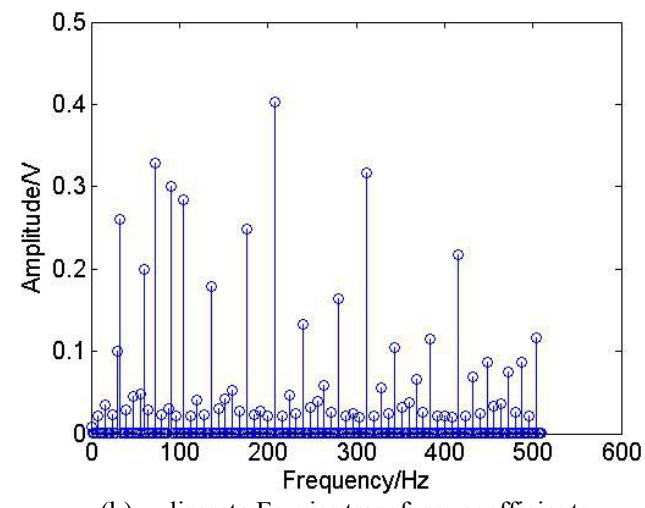

(b) discrete Fourier transform coefficients

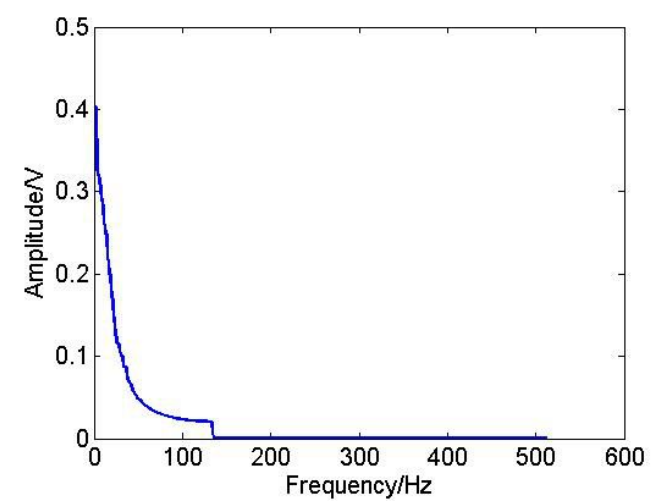

(c) The amplitude of Fourier transform coefficients as a function of freqrency

Figure 1. Fourier coefficients of vibration signal
The time domain waveform of vibration signal is given in Figure 1(a), the Fourier coefficients is illustrated in Figure 1(b), it can be seen from Figure 1(b) and Tab 1 that most of Fourier coefficients amplitude $(|f(\omega)|<3)$ are a small amount, the ordering result of Fourier coefficients amplitude is illustrated in Figure 1(c) which shows that coefficients decay and trend to zero in form of index. This explains that rolling bearing is a kind of approximately sparse signal in Fourier domain.

TABLE I. THE DISTRIBUTION OF FOURIER COEFFICIENTS

\begin{tabular}{ccc}
\hline $\begin{array}{c}\text { Fourier } \\
\text { coefficients } f(\omega)\end{array}$ & $|f(\omega)| \geq 3$ & $|f(\omega)|<3$ \\
\hline amount & 134 & 378 \\
\hline
\end{tabular}

\section{SIMULATION AND RESULTS}

The number of measurements $M$ is at least double to sparsity $k$, namely $M \geq 2 k$, sparsity $k$ is equal to 134 via analyzing sparsity of vibration signal, therefore, measurements $M$ is obtained by 268 , simulation measurements are divide into two parts in order to test and verify, one part is less than 268, namely 250 and 260 ,the other is greater than 268, namely a sequence of $268,270, \cdots, 290$, reconstruction algorithms is adopted by orthogonal matching pursuit.

Taking as an example of measurement matrix size $268 \times 512$, memory space of various measurement matrices is analyzed. Every entry of matrices need one space, namely 8bit, all entries of Gaussian random matrix are mutual independence, calculated mode of memory space is $268 \times 512 / 1024=134 \mathrm{~KB}$, Bernoulli random matrix is completely similar to Gaussian random matrix; Circulant matrix only needs one line of random elements based on its construction process, so its memory space is $512 / 1024=0.5 \mathrm{~KB}$, while Toeplitz matrix needs one line and one column of random entries so its memory space is $(268+512) / 1024=0.7617 \mathrm{~KB}$; Partially random Fourier matrix is obtained by randomly selecting deterministic Fourier matrix, so its memory space is $268 / 1024=0.2617 \mathrm{~KB}$, partially random Hadamard matrix is completely similar to partially random Fourier matrix. 


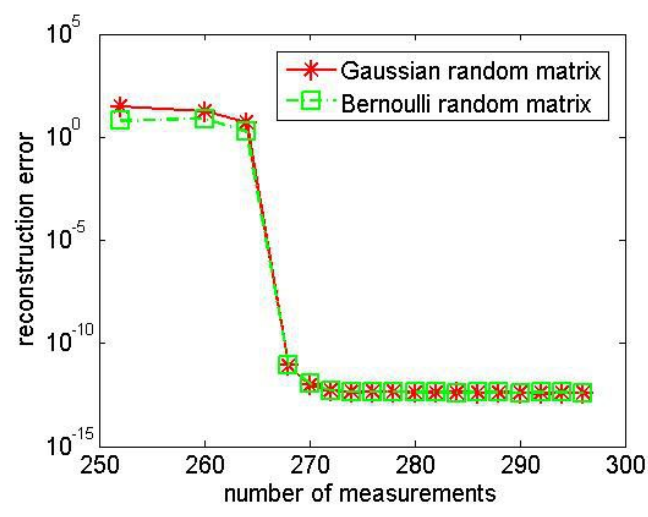

Figure 2. Reconstruction error as a function of number of measurements for random matrices

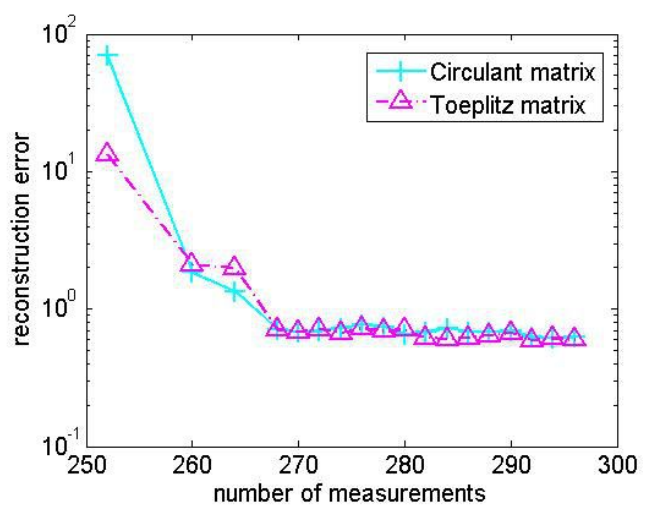

Figure 3. Reconstruction error as a function of number of measurements for deterministic matrices

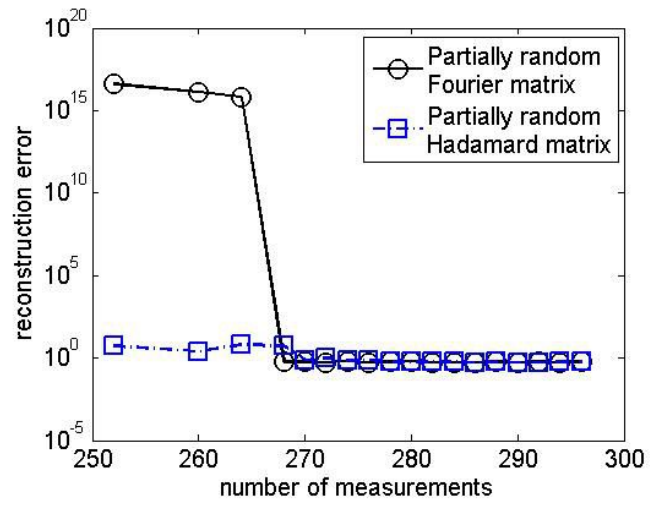

Figure 4. Reconstruction error as a function of number of measurements for partially random matrices

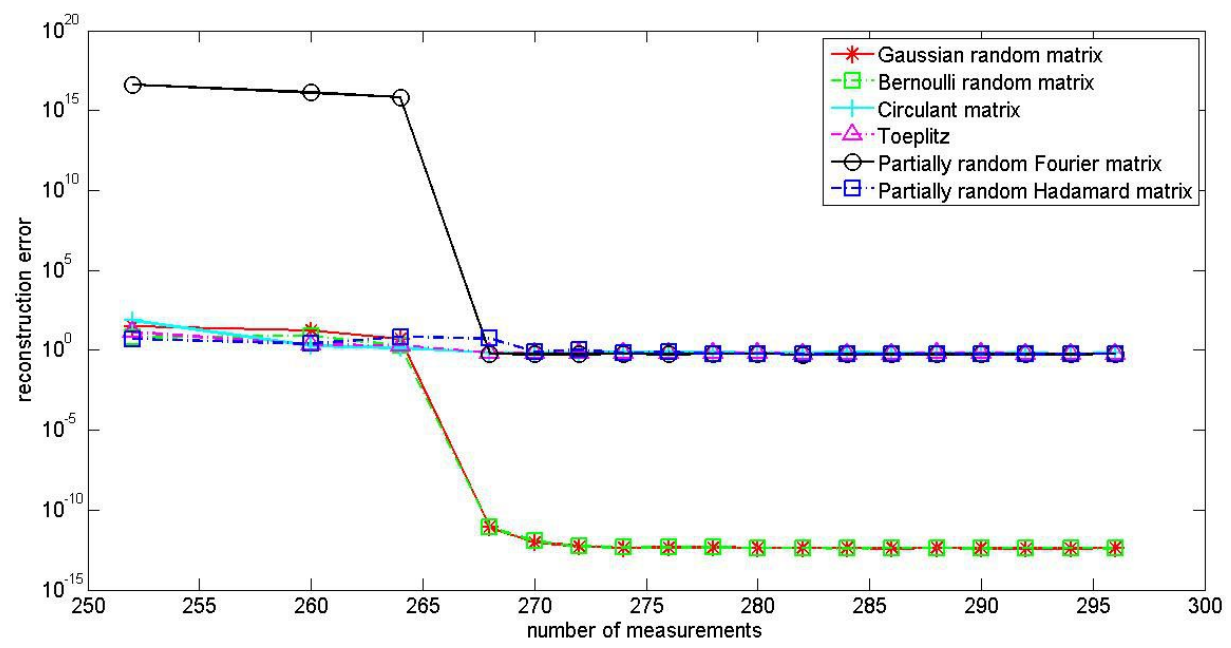

Figure 5. Reconstruction error as a function of number of measurements for common measurement matrices 
TABLE II. RECONSTRUCTION ERROR

\begin{tabular}{ccccccc}
\hline $\begin{array}{c}\text { Number of } \\
\text { measurements }\end{array}$ & 252 & 260 & 268 & 276 & 284 & 292 \\
\hline Gaussian matrix & $2.3992 \mathrm{e}+01$ & 4.7888 & $1.1375 \mathrm{e}-11$ & $4.4370 \mathrm{e}-13$ & $4.1585 \mathrm{e}-13$ & $3.9611 \mathrm{e}-13$ \\
Bernoulli matrix & 6.2390 & 7.7384 & $8.2815 \mathrm{e}-12$ & $4.5387 \mathrm{e}-13$ & $3.9057 \mathrm{e}-13$ & $4.2757 \mathrm{e}-13$ \\
Circulant matrix & $2.1505 \mathrm{e}+01$ & $2.1238 \mathrm{e}+01$ & 0.9324 & 0.6491 & 0.5676 & 0.7188 \\
Toeplitz matrix & $1.3196 \mathrm{e}+01$ & 2.1137 & $7.0710 \mathrm{e}-01$ & 0.7164 & 0.6060 & 0.5949 \\
$\begin{array}{c}\text { Partially Fourier } \\
\text { matrix }\end{array}$ & $4.1397 \mathrm{e}+16$ & $1.3082 \mathrm{e}+16$ & $5.9335 \mathrm{e}-01$ & 0.5405 & 0.5500 & 0.5673 \\
Partially Hadamard & 5.1945 & 2.4506 & 5.5204 & 0.7275 & 0.5766 & 0.5391 \\
\hline & & TABLE III. & MeMoRY sPACE & & Toeplitz & Partially \\
\hline Measurement matrices & Gaussian & Bernoulli & Circulant & Partially \\
\hline Memory space KB & 134.0000 & 134.0000 & 0.5000 & 0.7617 & 0.2617 & 0.2617 \\
\hline
\end{tabular}

It can be seen from Figure 5 and Tab 3 that the reconstruction error of common measurement matrices as the number of measurement increase overall, reconstruction error radically decrease when the number of measurement is equal to 268 because the number of measurement reach the reconstruction needed minimum amount. This also verifies that theoretical requested minimum measurement number. However, the decrease of reconstruction error is not apparent with the further increase of measurement number. Random measurement matrix compressed sensing mechanical vibration signal have exact reconstruction accuracy, but relatively need most memory space; Deterministic matrix and partially random matrix need fewer memory space, but do not have better reconstruction accuracy and sometimes even failure.

\section{CONCLUSION}

Adaptability of common measurement matrices for mechanical vibration signal is analyzed in this paper. The sensing performance of common measurement matrices for mechanical vibration signal is evaluated from the two perspective of reconstruction error and memory space. Two kinds of complete random matrices, Gaussian and Bernoulli matrices, can exactly reconstruct original vibration signal, but they occupy large memory space; Deterministic matrices, Circulant and Toeplitz matrices, alt-hough need fewer memory space, obtained measurements which do not have information of global vibration signal lead to lower reconstruction results; Partially random Fourier matrix is extremely coherent with sparse transforming base of vibration signal, so it has not exact result in the process of reconstructing original vibration signal, the requirement of Exponentiation of 2 seriously restrict its application.

This Project is supported by National Natural Science Foundation of China (Grant NO.51465034)

\section{REFERENCE}

[1] Cheng J, Yang Y, Yang Y. A rotating machinery fault diagnosis method based on local mean decomposition[J]. Digital Signal Processing, 2012, 22(2): 356-366.

[2] Bickson D, Baron D, Ihler A, et al. Fault identification via nonparametric belief propagation[J]. Signal Processing, IEEE Transactions on, 2011, 59(6): 2602-2613.

[3] Donoho D L. Compressed sensing[J]. Information Theory, IEEE Transactions on, 2006, 52(4): 1289-1306.

[4] Cohen A, Dahmen W, DeVore R. Compressed sensing and best $k$ term approximation[J]. Journal of the American mathematical society, 2009, 22(1): 211-231.

[5] Donoho D L, Elad M. Optimally sparse representation in general (nonorthogonal) dictionaries via $\ell 1$ minimization[J]. Proceedings of the National Academy of Sciences, 2003, 100(5): 2197-2202.

[6] Candes E J. The restricted isometry property and its implications for compressed sensing[J]. Comptes Rendus Mathematique, 2008, 346(9): 589-592.

[7] Tropp J, Gilbert A C. Signal recovery from partial information via orthogonal matching pursuit[J]. 2005.

[8] Foucart S, Rauhut H. A mathematical introduction to compressive sensing[M]. Basel: Birkhäuser, 2013.

[9] Candès E J. Compressive sampling[C]//Proceedings of the international congress of mathematicians:[s.n.] 2006, 3: 1433-1452.

[10] Candè E J, Wakin M B. An introduction to compressive sampling[J]. Signal Processing Magazine, IEEE, 2008, 25(2): 2130.

[11] Wang J. Support Recovery with Orthogonal Matching Pursuit in the Presence of Noise: A New Analysis[J]. arXiv preprint arXiv:1501.04817,2015. 